\title{
CHe CHAKIÑAN
}

Número 12 / DICIEMBRE, 2020 (18-31)

\section{Enoc Gutiérrez-Pallares}

egutierrezpa@uaemex.mx

Profesor de Tiempo Completo

Universidad Autónoma del Estado de

México, Unidad Académica Profesional

Cuautitlán Izcalli, Departamento de

Negocios Internacionales, Estado de

México, México.

ORCID:

http://orcid.org/0000-0002-3910-6289

\section{Miguel Ysrrael Ramírez- Sánchez}

miguel.ramirez@unini.edu.mx

Profesor Investigador Universidad

Internacional Iberoamericana,

Departamento de Doctorados.

Campeche, México

ORCID:

http://orcid.org/0000-0003-3045-5391

\section{Luis Manuel Borges-}

\section{Gouveia}

lmbg@ufp.edu.pt

Profesor de Tiempo Completo

Universidade Fernando Pessoa, Facultad de Ciencia y Tecnología, Departamento de Ciencias.

Porto, Portugal

ORCID:

http://orcid.org/0000-0002-2079-3234

Recibido:

(25/12/2019)

Aceptado:

$(23 / 04 / 2020)$

DOI:

https://doi.org/10.37135/chk.002.12.01

Artículo de Investigación 
Número 12 / DICIEMBRE, 2020 (18-31)

\section{CONSTRUCCIÓN DE UN MODELO EDUCATIVO A DISTANCIA CON FACTORES DE APRENDIZAJE Y PLATAFORMAS TECNOLOGICAS}

CONSTRUCTION
OF A DISTANCE
EDUCATIONAL MODEL
WITH LEARNING
FACTORS AND
TECHNOLOGICAL
PLATFORMS

\section{Resumen}

Este artículo se refiere a la importancia del crecimiento de la demanda educativa internacionalmente y los factores de aprendizaje en los modelos educativos modernos que presentan nuevos paradigmas, donde la Educación a Distancia $(\mathrm{EaD})$ emerge como una sólida respuesta para las universidades que deben garantizar pertinencia en la educación. Se presenta un diagnóstico cuantitativo de evaluación de formación a distancia, con un diseño experimental en referencia a las dimensiones pedagógico tecnológica como conjunto de variables independientes que integran las perspectivas y efectividad de enseñanza asistida por ordenador en la detección de necesidades educativas para el aprendizaje en línea del estudiante como variable de respuesta. Se tomó una muestra de $n=288$ personas integrada por diversos actores educativos y con base en la información obtenida se realiza un análisis de regresión lineal múltiple y aproximación factorial de componentes principales. El estudio permitió observar que la conducción de prácticas del aprendizaje a distancia, con respecto al aprendizaje de los estudiantes, requiere de un conjunto de distintas variables, en comparación con la enseñanza en el aula. La plataforma digital como soporte de las prácticas es considerada uno de los elementos principales, con impacto directo en la gestión de esta modalidad de educación.

Palabras clave: Aprendizaje en línea, pertinencia en la educación, educación a distancia, formación a distancia, enseñanza asistida por ordenador

\section{Abstract}

This article refers to the importance of the growth of educational demand internationally and the learning factors in modern educational models that present new paradigms, where Distance Education (DE) emerges as an essential response for universities that must guarantee relevance in education. Quantitative diagnosis of distance training evaluation is presented, with an experimental design about the technological pedagogical dimensions as a set of independent variables that integrate the perspectives and methodology of computer-assisted teaching in the detection of educational needs for learning student online as a response variable. A sample of $n=288$ people made up of various educational actors is required. Based on the obtained findings, multiple linear regression analysis and factor approximation of principal components were performed. The study related to the observation of the conduct of distance learning practices, concerning student learning, requires a set of different variables, compared to teaching in the classroom. The digital platform, as support for practices, is one of the main elements that directly impact the management of this type of education.

Keywords: Online learning, relevance in education, distance learning, distance learning, computer-assisted teaching. 


\section{INTRODUCCIÓN}

Cuando se hace referencia a los avances en educación, inmediatamente se vincula al acelerado desarrollo de la tecnología con nuevas formas de concebir el aprendizaje en las universidades. La educación superior debe distinguirse por características centrales que sean pilares de los requerimientos del contexto internacional, como la demanda social de ingreso (cada vez más amplia y diversificada), la incorporación de las Tecnologías de la Información y Comunicación (TIC) en los procesos de enseñanza-aprendizaje y la multiplicación de modelos de educación superior a distancia (Hernández 2017).

La incorporación de las TIC en las universidades ayuda a resolver el problema de la demanda educativa, pues permiten mayor cobertura con equidad (Zubieta \& Rama 2015), "salva las distancias geográficas entre los involucrados (institución, estudiantes y docentes) y atiende las diferencias sociales, económicas y culturales" (Didou 2014; Moreno 2015, citados por Luna, Ponce, Cordero \& Cisneros 2018:2).

En definitiva, para poder ofertar y asegurar pertinencia de la educación, debe mostrarse una articulación coherente y armónica de un modelo que, por encima de los instrumentos, privilegie el sentido pedagógico de los procesos entendiendo que "una educación de calidad puede salir adelante con una tecnología inadecuada; pero jamás una tecnología excelente podrá sacar adelante un proceso educativo de baja calidad" (Mestre y Ruíz 2013:19).

En ese sentido, es preeminente que las personas que estudien en la modalidad no escolarizada y a distancia sean formadas enelámbito del aprendizaje y por medio de la plataforma educativa, tengan a bien disposición por la adaptabilidad a los sistemas y formas de aprendizaje con medios tecnológicos, desarrollo de capacidades y habilidades que se conviertan en competencias para el desarrollo del campo laboral, asegurando éxito en el desempeño profesional y para la vida (García, Ayestarán, López

\section{\& Tovar 2019)}

Existe una diferencia significativa entre planear una clase para el modelo presencial tradicional y aquella clase en la que implique un ambiente de aprendizaje con una amplia concepción de recursos y la programación de actividades en el ámbito virtual, que integre la elaboración de guías que conduzcan al autoaprendizaje y el autodidactismo por parte de los estudiantes y con el apoyo y guía del docente (Vargas 2015).

Para Molina et al. (2015), el componente tecnológico se debe asociar al sistema de comunicación mediada por ordenador y vincularlo con los actores del proceso pedagógico de Enseñanza-Aprendizaje, basado "en la comunicación de forma multidireccional, de manera que cada estudiante participe de forma activa y autónoma" (Saza-Garzón 2016:107), con un "sistema de aprendizaje flexible, dinámico y adaptativa al medio donde se desarrolla" (Juca 2016:107), de recursos compartidos, estabilidad, comodidad y apoyo específico a la actividad del grupo.

Las Instituciones de Educación Superior (IES) han realizado el compromiso de garantizar calidad educativa en la Educación a distancia (EaD), sin embargo, los nuevos paradigmas en esta modalidad de educación son verdaderos retos en las correlaciones de las dimensiones organizativa, académica y tecnológica, en la cual cada una ha mostrado sus avances con las experiencias adquiridas en el inicio de estas modalidades, teniendo como un tema central el aprendizaje del estudiante por medio de una plataforma educativa como variable de estudio.

Cuando referimos a la EaD y al aprendizaje como variable de estudio, existe una problemática sobre las dimensiones pedagógico tecnológicas con relación al modelo educativo que opera en cada IES, diversos estudios cuantitativos (Gallego \& Araque 2019; Arancibia, Cabrero \& Marin 2020; Pulido, Rodríguez, Santana \& Lorenzo 2020; Parra 2015; Han \& Ellis 2020; Ramos, García, Sotelo, López \& Murillo 2020; García, López \& Cabero 2020; Fernández, García \& López 2015), muestran el interés de investigar las correlaciones entre las variables de influencia, estrategias y factores, que 
apoyados en técnicas estadísticas como el análisis factorial y extracción de componentes principales permiten identificar aquellas que muestran la mejor aportación en el aprendizaje del estudiante como variable de respuesta.

En la evaluación de un modelo educativo, la metodología del que parte la mayoría en sus procesos de enseñanza aprendizaje corresponde a procesos lineales de diseño didáctico e instruccional de forma interactiva y secuencial (Belloch 2013), la metodología ADDIE (Análisis, Diseño, Desarrollo, Implementación y Evaluación) (Perch, Prieto, García \& Orozco 2019) es la que se ha elegido para el estudio del modelo, mostrando la primera etapa para desarrollar la detección de necesidades.

Atendiendo a la importancia del estudio del aprendizaje del estudiante, en este trabajo se presenta la evaluación de un modelo de $\mathrm{EaD}$ en la Universidad Autónoma del Estado de México en su plataforma educativa SEDUCA, en tres programas educativos ofertados en la modalidad no escolarizada y a distancia en la Unidad Académica Profesional de Cuautitlán Izcalli (UAPCI), del cual se toma una muestra de 288 personas que representa a todos los actores educativos implicados.

Se realiza un análisis de correlación de variables de estudio con un conjunto de 6 variables en el instrumento aplicado como variables independientes y que dan respuesta a la variable dependiente que es el aprendizaje del estudiante, posteriormente se aplica un análisis factorial y de extracción de componentes principales para mostrar los resultados con la transformación del conjunto de variables con mayor impacto. El aporte de la investigación se evidencia en mostrar la importancia de un adecuado diseño instruccional en la plataforma educativa utilizada, así como que en $\mathrm{EaD}$ se requiere un conjunto distinto de variables en comparación con la enseñanza tradicional.

\section{METODOLOGÍA}

Este trabajo se alinea dentro del paradigma de la investigación cuantitativa, de diseño transversal con enfoque descriptivo correlacional debido a que se indaga el aprendizaje de los estudiantes como variable dependiente a través de la percepción que se tiene en la modalidad no escolarizada y el modelo que opera, concentrado en un conjunto de 6 variables en un cuestionario de 51 ítems.

La participación del estudio concentra a todos los actores educativos involucrados en el proceso con una muestra de tipo intencional que sigue los criterios del muestreo probabilístico aleatorio simple (Cerón 2006) de 288 personas con tres generaciones de alumnos, contemplando a diseñadores de materiales, asesores, tutores, profesores, gestión académica pertinente, infraestructura tecnológica, entre otros.

Las mediciones se realizaron para evaluar la correlación existente entre las variables que favorecen las capacidades de aprendizaje en los estudiantes, utilizando técnicas estadísticas de tipo descriptivas, correlacionales, factorial y de transformación de variables para la extracción de componentes principales, analizando las problemáticas existentes respecto a la cimentación de un modelo que muestre control, actualización y adaptabilidad en los procesos de aprendizaje con materiales integrados y mediados por las TIC, que muestren Entornos Virtuales de Aprendizaje (EVA) para el estudiante (Calzada, Maceo \& Bennasar 2016) tomando en cuenta necesidades, actividades y recursos que deben desarrollarse para lograrlo.

En la descripción y análisis "se responde con una pruebaque refiere aunestudioenelque se manipulan intencionalmente las variables independientes, con la finalidad de analizar las consecuencias que tiene sobre la variable dependiente y para analizar sus posibles resultados" (Hernández, Fernández \& Baptista 2014:129), con el cual se busca comprender una evaluación de la situación actual del modelo educativo con la plataforma. 
El estudio se realiza por medio de un cuestionario integrado por 51 ítems y que concentra un conjunto de 6 categorías como variables, a saber:

1. el diseño curricular y sus aspectos fundamentales para el aprendizaje,

2. objetivos del plan de estudios y áreas disciplinares,

3. conocimientos previos,

4. la enseñanza, que contempla diseño de material, calidad, presentación, actividades y diversos sub-elementos que conforman la variable como conjunto,

5. la evaluación y seguimiento y

6. el funcionamiento de la plataforma educativa, dando respuesta a la medición del aprendizaje de los estudiantes como variable de resultado y grado de satisfacción.

Una vez que se llevó a cabo la recolección de datos, los diferentes análisis y tratamiento de los datos se han realizado con el programa estadístico SPSS 24.0. Se realizó un estudio correlacional del conjunto de variables; mostrando su aportación a la variable de respuesta, de igual forma se analizan los errores de predicción con el coeficiente de DurbinWatson y análisis ANOVA para la no contradicción entre el conjunto de variables con intervalos de confianza que muestran la aceptabilidad de las pruebas y tratamiento de datos realizados.

En la metodología ADDIE se infiere que se establezcan premisas de carácter general en los modelos educativos a distancia, como su primera fase lo indica, en un análisis y diagnóstico se realiza la propuesta de una proyección de un modelo de regresión lineal múltiple, que a través de la transformación de variables en factores de componentes principales se extraigan los elementos primarios, con el objetivo de dar respuesta al aprendizaje del estudiante, con aportación del contexto mencionado y que pueda ser un referente para otras IES.

\section{RESULTADOS Y DISCUSIÓN}

El diagnóstico se llevó a cabo con base en tres programas educativos que se ofertan para la modalidad no escolarizada y a distancia en la UAPCI: Negocios Internacionales, Logística y Derecho Internacional. Dichos programas se iniciaron en 2013, fundamentados en los estudios de factibilidad de la región IV del Estado de México, con el propósito de brindar educación no escolarizada de calidad, innovadora y contextualizada por medio de la plataforma educativa institucional denominada SEDUCA, de la Universidad Autónoma del Estado de México (Universidad Autónoma del Estado de México [UAEM] 2010).

Se tomó una muestra de 288 personas considerando aquellos actores educativos que están emergidos en la operatividad del modelo, de los cuales el $43.1 \%$ son hombres, $56.9 \%$ mujeres; la participación por programa educativo fue de $27.9 \%$ para Negocios Internacionales, $16.6 \%$ para Logística y $55.5 \%$ para Derecho Internacional, con un rango de edad entre los 18 a los 60 años y una media de 34 años.

El diagnóstico realizado a través del cuestionario recogió información significativa en torno al grado de operatividad y eficiencia de la plataforma educativa, el aula virtual, y tomando referencia el estado de las tres dimensiones que operan en los programas educativos para esta modalidad. Las variables consideradas que se establecieron en el estudio para dicha aportación fueron: diseño instruccional, objetivos del programa educativo y de las Unidades de Aprendizaje, conocimientos previos, la enseñanza, evaluación y las generalidades del modelo SEDUCA, dando respuesta a la variable aprendizaje y satisfacción del estudiante.

La información recolectada parte de la base de una matriz de 51 valores contemplados en una escala de evaluación de 1 a 10 por 288 miembros de la muestra (matriz $288 \times 51,14688$ valores totales), 
contemplando la agrupación y categorizaron de los ítems en 6 grupos de variables que brindan aportación al aprendizaje en el estudiante. El tratamiento de los datos se llevó a cabo por medio de la concentración de las respuestas obtenidas en los ítems de cada muestra y agrupándolo por conjunto de variables; los análisis se llevaron a cabo por medio del software estadístico SPSS versión 24.0. En la tabla 1 se muestra la concentración del conjunto de variables consideradas para el aprendizaje del estudiante, cada conjunto categórico de las variables se compone alrededor de 8 a 9 ítems del cuestionario, en la cual se tiene la matriz inicial de correlación que son consideradas para el modelo de regresión lineal múltiple.

Tabla 1: Variables de entradas/eliminadas consideradas en el modelo de regresión múltiple para el aprendizaje

\begin{tabular}{|c|c|c|c|}
\hline Modelo & Variables entradas & $\begin{array}{c}\text { Variables } \\
\text { eliminadas }\end{array}$ & Método \\
\hline 1 & DiseñoInstruccional $\left.\right|^{b}$ & & Entrar \\
\hline 2 & Objetivos $^{b}$ & & Entrar \\
\hline 3 & ConocimientosPrev ${ }^{b}$ & & Entrar \\
\hline 4 & Enseñanza $^{b}$ & & Entrar \\
\hline 5 & Evaluacion $^{b}$ & & Entrar \\
\hline 6 & Plataforma $^{b}$ & & Entrar \\
\hline
\end{tabular}

a. Variable dependiente: Aprendizaje

b. Todas las variables solicitadas introducidas.

Fuente: Elaboración propia

\section{SOBRE LAS REGIONES GEOGRÁFICAS}

Los resultados en torno a los alcances geográficos con respecto de la demanda educativa por parte de la comunidad estudiantil y del modelo no escolarizado a distancia se presentan en los ámbitos internacionales, con estudiantes de Estados Unidos y Colombia, y para el ámbito nacional en los estados de Quintana Roo, Michoacán, San Luis Potosí, Querétaro y del Estado de México. Al ser la muestra que integra la comunidad de actores educativos en la modalidad no escolarizada, se parte de la observación y experiencia brindadas en las generaciones de estudiantes hasta el momento y se estima que esta muestra pueda inferir y mostrar aportaciones al aprendizaje para contextos de estudio similares a nivel internacional.

\section{SOBRE LAS VARIABLES PREDICTORAS INICIALES}

Una vez concentrada la información en el software de paquetería estadística, se muestra el proceso de segmentación y progresión de aportación de cada conjunto de las variables descritas por la matriz de 51 valores y que relaciona directamente a la variable dependiente aprendizaje, se inicia con un valor de 0.225 para el factor $\mathrm{R}$ cuadrado, lo que indica la aportación de la primera variable a la aproximación lineal del modelo, el conjunto de variables va mostrando su aportación una a una, hasta llegar a un valor de 0.38 , lo cual implica la relación, adición y aportación que cada variable aporta al modelo de aprendizaje.

En la tabla 2 se muestra el resumen y aportación del conjunto de valores de las variables predictores iniciales, el coeficiente de Durbin-Watson (C.D. $\mathrm{W}<2$ ) muestra precisión al tener su valor por debajo de 2, en los errores de predicción de la regresión, por lo que se deduce que el conjunto de valores integrados, si están aportando la tendencia de una correlación lineal múltiple para el establecimiento de un modelo para el aprendizaje del estudiante con el conjunto de variables consideradas.

De igual forma se realiza un análisis ANOVA para cada variable, donde se hace manifiesto con valor de significancia menor a 0.001 (pues el intervalo de confianza fue del 99\%) lo que da muestra de que ninguna variable entra en contradicción de una correlación incongruente entre ellas. 
Tabla 2: Aportaciones promedio del conjunto de variables predictoras

\begin{tabular}{l|c|c|c|c|c} 
Modelo & $\mathrm{R}$ & $\mathrm{R}$ cuadrado & $\begin{array}{c}\text { R cuadrado } \\
\text { ajustado }\end{array}$ & $\begin{array}{c}\text { Error estándar de la } \\
\text { estimación }\end{array}$ & Durbin-Watson \\
\hline 1 & $.474^{\mathrm{a}}$ & .225 & .222 & 1.07863 & \\
\hline 2 & $.474^{\mathrm{b}}$ & .225 & .220 & 1.08042 & \\
\hline 3 & $.543^{\mathrm{c}}$ & .295 & .288 & 1.03218 & \\
\hline 4 & $.609^{\mathrm{d}}$ & .371 & .362 & .97702 & \\
\hline 5 & $.613^{\mathrm{e}}$ & .376 & .365 & .97442 & \\
\hline 6 & $.617^{\mathrm{f}}$ & .380 & .367 & .97290 & 1.981 \\
\hline
\end{tabular}

a. Predictores: (Constante), Diseño Instruccional

b. Predictores: (Constante), Diseño Instruccional, Objetivos

c. Predictores: (Constante), Diseño Instruccional, Objetivos, Conocimientos Prev

d. Predictores: (Constante), Diseño Instruccional, Objetivos, ConocimientosPrev, Enseñanza

e. Predictores: (Constante), Diseño Instruccional, Objetivos, ConocimientosPrev, Enseñanza, Evaluación

f. Predictores: (Constante), Diseño Instruccional, Objetivos, ConocimientosPrev, Enseñanza, Evaluación, Plataforma

g. Variable dependiente: Aprendizaje

Fuente: Elaboración propia

En el campo educativo se mencionan las curvas de aprendizaje como factor de adaptabilidad de los seres humanos para tener percepción y significancia en las experimentaciones de lo que sucede alrededor, se hace referencia a informes aplicados a la industria con procesos de ensamblaje de aviones, a partir de la consideración de diversos factores que influyen en el aprendizaje de una persona en la industria con el desarrollo de competencias profesionales y para la vida; de igual forma se generan límites de control para los procesos productivos en las áreas manufacturera y organizativa (Rodríguez 2016).

Por lo tanto, una curva de aprendizaje no es más que una línea que muestra la relación existente entre un conjunto de dos variables y la relación de ellas siendo dependiente una de otra, considerando variables fundamentales con la medición de tiempo y teniendo como base el proceso pedagógico de adaptabildiad y asimiliación del conocimiento, se busca que la curva de aprendizaje se pueda linealizar por los múltiples factores en su consideración.

En este trabajo lo que se pretende no es versar sobre la curva de aprendizaje, sino más bien realizar la propuesta de la aproximación lineal de un modelo que a través de la consideración del conjunto de varibales mencionadas y con la exploración de ellas en la extracción de componentes principales, se proponga con variables múltiples la generalización de un modelo que prediga la aportación al aprendizaje del estudiante en la modalidad a distancia y con ello asegurar la pertinencia de la educación. 


\section{TRANSFORMACIÓN DE VARIABLES PREDICTORAS EN COMPONENTES PRINCIPALES}

Una vez realizada la prueba estadística de factores de correlación y aportaciones para el conjunto de variables, con la finalidad de mejorar la correlación del conjunto obtenido y aportaciones en el coeficiente $\mathrm{R}$ cuadrado de linealidad, se lleva a cabo una transformación de variables con los valores de la matriz concentrada, la idea es extraer un conjunto de valores implícito de cada conjunto, $\mathrm{y}$ encontrar aquel subconjunto de variables transformadas con la extracción de factores que muestren aportación al estado de eficiencia para el aprendizaje del estudiante y evaluando qué componentes principales son los que aportan significancia para la propuesta de integración, y cuáles no, pero que aun así aportan criterio de valor y resulta necesario que se encuentren en la construcción como elementos en el modelo.

Para llevar a cabo el Análisis Factorial (AF) se realiza una transformación de variables, tomando el conjunto de variables independientes y la variable de relación aprendizaje del estudiante. Esta matriz de valores contiene implícitamente una serie de subvariables denominadas variables latentes (no observables), y que llevando a cabo el AF, se obtiene el conjunto de variables latentes que serán los factores de aportación en la regresión lineal múltiple. Este conjunto obtenido es reducido al fundamental, y dan reflejo de una relación adecuada para los modelos de regresión lineal y de correlaciones parciales.

Como primera instancia se escalan y ponderan los valores de los subvalores para cada variable, el conjunto de variables es de 6 en total, se realiza una correlación de las variables y la construcción de un modelo de regresión lineal múltiple con un intervalo de confianza del $99 \%$, la relación de variables se realizó de acuerdo en el orden establecido en el instrumento, como se muestra en la tabla 1, con estimaciones y ajuste al modelo y diagnósticos de colinealidad.

Una vez realizado, se infiere sobre el estado actual del modelo educativo con base en el aprendizaje de los estudiantes y la recolección de los datos de los factores que muestran significancia. Dado que la investigación se muestra como un diagnóstico del estado de eficiencia en relación con el aprendizaje, este refleja la situación en el que el modelo educativo operante se encuentra, es decir, la relación directa entre el grado de satisfacción con base en el aprendizaje del estudiante.

Para profundizar en el estudio existe la extracción de aportación de significancia de cada subvalor que conforma el conjunto de variables, se realizó un análisis factorial de los elementos como técnica de escalamiento y con el propósito de mostrar la aportación significativa de los elementos, se encuentran los subelementos pilares de la variable que pueden representarla en una transformación de componentes primarios que describan a la variable por encima de 0.50 de su aportación del modelo general y de medias.

Cada subconjunto de valores transformado en la aportación de factores, es el nuevo conjunto de elementos para fundamentar el modelo de regresión lineal múltiple de componentes principales, esperando que el reflejo de cada subvalor incremente los valores del coeficiente $R$ cuadrado general e integrado un valor de 0.504, como lo muestra la tabla 3, sobre el sub-conjunto de valores, que indica que en la extracción de esos subvalores se puede encontrar el comportamiento lineal múltiple para el aprendizaje del estudiante. 
Tabla 3: Aportaciones de subvalores con variables y conjunto de factores

\begin{tabular}{l|c|c|c|c}
\multicolumn{1}{c}{ Modelo } & $\mathrm{R}$ & $\mathrm{R}$ cuadrado & $\mathrm{R}$ cuadrado ajustado & $\begin{array}{c}\text { Error estándar de la } \\
\text { estimación }\end{array}$ \\
\hline $\begin{array}{l}\text { 1 Diseño } \\
\text { Instruccional }\end{array}$ & $.520^{\mathrm{a}}$ & .271 & .266 & 1.04822 \\
\hline 2 Objetivos & $.594^{\mathrm{b}}$ & .353 & .344 & .99088 \\
\hline $\begin{array}{l}\text { 3 Conocimientos } \\
\text { Previos }\end{array}$ & $.639^{\mathrm{c}}$ & .408 & .396 & .95070 \\
\hline 4 Enseñanza & $.687^{\mathrm{d}}$ & .472 & .457 & .90114 \\
\hline 5 Evaluación & $.710^{\mathrm{e}}$ & .504 & .485 & .87768 \\
\hline $\begin{array}{l}\text { 6 Plataforma } \\
\text { educativa }\end{array}$ & $.710^{\mathrm{f}}$ & .504 & .483 & .87912 \\
\hline
\end{tabular}

a. Predictores: (Constante), Factores de Diseño Instruccional

b. Predictores: (Constante), Factores de Diseño Instruccional, Factores de Objetivos

c. Predictores: (Constante), Factores de Diseño Instruccional, Factores de Objetivos, Factores Conocimientos previos

d. Predictores: (Constante), Factores de Diseño Instruccional, Factores de Objetivos, Factores conocimientos previos, Factores de Aprendizaje

e. Predictores: (Constante), Factores de Diseño Instruccional, Factores de Objetivos, Factores conocimientos previos, Factores de Aprendizaje, Factores de evaluación

f. Predictores: (Constante), Factores de Diseño Instruccional, Factores de Objetivos, Factores conocimientos previos, Factores de Aprendizaje, Factores de plataforma educativa

Fuente: Elaboración propia

En la transformación y propuesta del modelo bajo la perspectiva de componentes principales se muestra un valor mayor que el modelo base, de 0.22 a 0.27 , es decir, la extracción de los componentes en comparativa con el modelo base muestra riqueza por medio de la transformación de factores, que implica que a primera instancia puede no representar linealidad para el aprendizaje, pero es precisamente en la extracción que el modelo toma sentido para su propuesta.

De igual forma, el modelo de regresión lineal múltiple indica que los conjuntos de aportaciones de los factores muestran un coeficiente de correlación R cuadrado de 0.978 , como lo muestra la tabla 4, con el cual la linealidad del modelo para el aprendizaje se puede mostrar a partir de la extracción de factores y hacer viable la propuesta del modelo por medio del AF y la transformación de valores en componentes principales.
En la propuesta de linealización del aprendizaje debemosentenderque laextracción de componentes es la forma de reflejar los componentes que brindan respuesta a la aportación de aquellos elementos que deben integrar la propuesta de un modelo de EaD y que deben ser fundamentales, pues son ellos los que brindarán aportaciones y de esta manera garantizarán pertinencia de la educación dentro de las plataformas educativas y dando cuenta de los elementos fundamentales para su construcción, existiendo algunos como la plataforma educativa que parece no aportar al modelo, pero que es necesaria que esté presente para el tipo de modalidad no escolarizada. 
Tabla 4: Resumen del modelo con extracción de factores

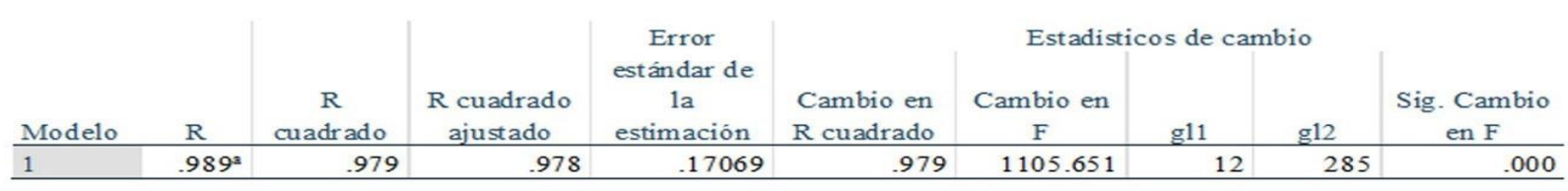

a. Predictores: (Constante), REGR factor score 1 for analysis 2, REGR factor score 2 for analysis 1 , REGR factor score 2 for analysis 1, REGR factor score 3 for analysis 1, REGR factor score 1 for analysis 1, REGR factor score 2 for analysis 1, REGR factor score 2 for analysis 1, REGR factor score 2 for analysis 1 , REGR factor score 1 for analysis 1 , REGR factor score 1 for analysis 1 , REGR factor score 1 for analysis 1, REGR factor score 1 for analysis 1

b. Variable dependiente: Enseñanza

Fuente: Elaboración propia

\section{GENERALIDADES DEL MODELO EDUCATIVO SEDUCA}

Como último apartado, dentro del estudio de diagnóstico y parte del instrumento realizado a la población participante en la comunidad de la plataforma educativa estudiada (SEDUCA) sobre el modelo educativo, se cuestionó sobre indicadores directos relacionados con la operatividad y gestión. La tabla 5 muestra las generalidades de la plataforma educativa SEDUCA como evaluación diagnóstica donde se exponen los conceptos, la califación global obtenida en la escala de 1 al 10, así como las observaciones principales de los extractos del conjunto de componentes y variables obtenidas en la recogida de datos.

La investigación se ha realizado con la comunidad que compone la plataforma educativa de la modalidad no escolarizada del espacio académico de Cuautitlán Izcalli, con la oferta de las licenciaturas. La muestra aporta el alcance que tiene la oferta de $\mathrm{EaD}$ con las regiones a las cuales se tiene cobertura, con la finalidad de conocer la eficiencia del modelo educativo que se lleva a cabo para el aprendizaje y la proyección de un modelo que incluya diversos factores de aprendizaje que integren a su vez procedimientos que rompan la brecha entre un modelo tradicional de la impartición de los procesos de enseñanzaaprendizaje en la EaD.

El estudio general dimensiona una matriz de 288 x 51 elementos en 6 conjuntos de variables, la idea general del estado de eficiencia del modelo educativo que rige en el espacio académico que considera solo estos elementos, da muestra sobre el crecimiento en el aprendizaje en línea, y muestra una relación lineal con el incremento de elementos que aportan significancia y que pueden incluirse aquellos considerados imprescindibles para este tipo de educación.

Dentro de los resultados expresados se hallan áreas de oportunidad en los textos e información vertidos en las Guías de Estudio Independiente que son el aula de clases del estudiante, a través de la presentación de la información y diseño de actividades. Es relevante hacer énfasis en la existencia de una alta correlación que refleje el grado de aprovechamiento y aprendizaje en el estudiante.

Maldonado y López (2019) afirman que uno de los mayores retos de la $\mathrm{EaD}$ es producir materiales dentro de la plataforma educativa que promuevan y estimulen el aprendizaje de los estudiantes, es decir, deben incluir actividades que los ayuden a entender, desarrollar y practicar habilidades para aprender. 
Tabla 5: Generalidades del Sistema SEDUCA, evaluación diagnóstica

\begin{tabular}{|c|c|c|}
\hline \multicolumn{3}{|c|}{ Calidad en la plataforma SEDUCA en relación a: } \\
\hline Concepto & Calificación & Observaciones \\
\hline Materiales expu estos para el estudio & 8.7 & Contenidos en las GEI mostrados a través de la plataforma \\
\hline Actividades diseñadas para el aprendizaje & 8.6 & Comprende Foros, Portafolios, Wikis, Chat, Aula virtual etc. \\
\hline Atención dedicada a los estudiantes & 8.5 & $\begin{array}{l}\text { Comunicación principalmente con el profesor, coordinador y la } \\
\text { administración escolar. }\end{array}$ \\
\hline Gestión académica & 7.8 & $\begin{array}{l}\text { Procesos de control escolar (inscripciones, constancias, exámenes, } \\
\text { calificaciones etc.). }\end{array}$ \\
\hline Evaluación realizada & 8.2 & $\begin{array}{c}\text { Estructura y forma de llevar a cabo la evaluación a través de las } \\
\text { diversas actividades }\end{array}$ \\
\hline $\begin{array}{c}\text { Tutorización (Consideran que debe } \\
\text { existir) }\end{array}$ & $50.4 \%$ & Tutor en linea (aún no existe) \\
\hline Plataforma flexible & 8.5 & \\
\hline $\begin{array}{l}\text { Inversión de tiempo en la realización de } \\
\text { actividades }\end{array}$ & $\begin{array}{c}53.5 \% \text { más } \\
\text { de } 3 \text { horas } \\
42.8 \% \text { de } 2- \\
3 \text { horas } \\
3.7 \% \text { a lo } \\
\text { más una } \\
\text { hora }\end{array}$ & $\begin{array}{l}\text { Tiempo que dedica el estudiante a una actividad de una sola } \\
\text { Unidad de Aprendizaje }\end{array}$ \\
\hline $\begin{array}{l}\text { Evaluación por parte de los profesores } \\
\text { hacia los estudiantes }\end{array}$ & 8.0 & $\begin{array}{l}\text { La retroalimentación por parte de los profesores se considera un } \\
\text { aspecto que se debe atender. }\end{array}$ \\
\hline Atención administrativa & 7.9 & $\begin{array}{l}\text { Tramites diversos pertenecientes al área administrativa (becas, } \\
\text { servicio social, practica profesionales, entre otras). }\end{array}$ \\
\hline
\end{tabular}

Fuente: Elaboración propia

Los análisis estadísticos son una consideración significativa del reflejo de un modelo educativo presencial que atraviesa una transición, lainferencia es hasta cierto punto normal porque para quienes diseñan materiales, los profesores, "plantea un reto para las instituciones, especialmente las universidades siendo utilizada por muchas organizaciones educativas sin garantizar el cumplimiento de calidad mínima exigida“ (Baldomero, Vázquez \& Belando 2018:74); y en los profesores como función de diseñadores, quienes están sumidos en su formación y práctica docente, pero de manera presencial.

Podemos entonces entender que la universidad necesita capacitar y emprender acciones para establecer un modelo que muestre procedimientos, integre fundamentaciones pedagógicas y teorías contemporáneas que garanticen un modelo con sustento en las TIC, para el desarrollo de aprendizajes y comunicación de forma que se puedan construir conocimientos en los estudiantes a través de Entornos Virtuales Personales de
Aprendizaje (PLE) y el dominio, contextualización y operatividad del aula de clases virtual (Goodyear \& Dimitriadis 2013).

El estudio diagnóstico nos muestra claramente la oportunidad de poder mejorar el establecimiento de rúbricas para las actividades diseñadas, los tiempos de respuesta de evaluación de estas, la cantidad de actividades diseñadas y su grado de complejidad. De igual forma, se quiere evitar en todo instante la impresión de un modelo presencial plasmado en una plataforma virtual.

Es un reto poder entablar la propuesta en relación con la correlación de variables y linealidad múltiple del modelo, sin embargo, este diagnóstico fundamentará las bases para la construcción del modelo pedagógico didáctico que se quiere para los programas educativos de la modalidad no escolarizada del espacio académico, con elementos de aportación significativa por factores y escalamiento de aportación de elementos, conjuntos, subconjuntos y aportaciones a la 
comunidad científico-académica que vincula sus investigaciones a la $\mathrm{EaD}$, en función de llevar a cabo formas y metodologías similares para la evaluación de sus modelos educativos.

\section{CONCLUSIONES}

Este trabajo aporta una visión general de los aspectos más relevantes de un modelo de $\mathrm{EaD}$ que por medio de la consideración de componentes iniciales fundamentales en el tipo de educación busca complementar y dar solución a los pilares de la educación tradicional, factores fundamentales como la presencialidad y la distancia deben ser acotados por la transformación de modelos que den respuesta a los paradigmas de la nuevaeducación.

La intención del objetivo del trabajo ha sido fundamentar una propuesta de aprendizaje desde la linealización de múltiples variables, estableciendo bases para la construcción del modelo pedagógico didáctico que se quiere en los programas educativos de la modalidad no escolarizada, con elementos de aportación significativa por medio de la transformación de variables y con ello, aportar a la comunidad científico-académica que vincula sus investigaciones a la $\mathrm{EaD}$, para que se puedan llevar a cabo formas y metodologías similares en función de la evaluación de sus modelos educativos.

La detección del diagnóstico de necesidades y del estado del modelo en los resultados presentados hace pertinente la creación de escenarios que garanticen el aprendizaje, que muestren adaptabilidad en su desarrollo e implementación de manera óptima y creciente, y que además se fortalezcan en la gestión y administración escolar, la comunicación efectiva y en el uso dinámico de diseño de Entornos Virtuales de Aprendizaje (EVA), que brinden aportación por encima de un modelo presencial reflejado en una plataforma.

Los extractos factoriales y conjuntos de subvalores de cada elemento de la matriz muestran aportación significativa, con la atención en los elementos y factores de aprendizaje en la modalidad, se pueden construir elementos de integración cada vez más complejos, haciendo que sean considerados para robustecer la composición del modelo y su linealización.

La propuesta para la integración de los elementos que aportan significancia en el modelo es importante, aquellos que no la muestran deben ser atendidos como área de oportunidad, para que de esta manera muestren su aportación en adaptaciones futuras realizadas al modelo que opera actualmente; considerando que para el diseño de un modelo didáctico pedagógico a distancia deben responder en todo momento a las necesidades educativas.

En los resultados obtenidos se deja evidencia clara de futuras investigaciones en la construcción de modelos de diseño instruccional y didácticos en sus componentes principales de integración, que muestren el estado de evidencia al ser evaluados por un diagnóstico y que ofrezcan una propuesta de integración de elementos en un modelo lineal múltiple y con curvas de aprendizaje, que pueda garantizar la atención de las necesidades, ser flexible, adaptable y evaluable.

\section{DECLARACIÓN DE CONFLICTOS DE INTERESES:}

Los autores declaran no tener conflictos de interés.

\section{REFERENCIAS BIBLIOGRÁFICAS}

Arancibia, M. L., Cabrero, J. I. \& Marin, V. (2020). Análisis factorial de una escala de creencias sobre la enseñanza y su relación con características personales y profesionales de docentes de Educación Superior. Revista ESPACIOS, 41(2), 1-7.

Baldomero, M., Vázquez-Cano, E. \& BelandoMontoro, M. (2018). Diseño de un modelo de evaluación de la calidad de los cursos MOOC mediante Lógica difusa. Revista 
Electrónica de Investigación Educativa, 20(4), 72-85. doi:https://doi.org/10.24320/ redie.2018.20.4.1663

Belloch, C. (2013). Modelo ADDIE. Entornos Virtuales de Formación. Universitat de València. Recuperado de http://www.uv.es/ bellochc/pedagogia/EVA4.wiki?7

Calzada, A. H., Maceo, Y.C. \& Bennasar, F. N. (2016). Diagnóstico de necesidades y uso de las TIC para la evaluación del aprendizaje en física en la Universidad de las Ciencias Informáticas. EDUTEC. Revista Electrónica de Tecnología Educativa, (55), a326.doi: https://doi. org/10.21556/edutec.2016.55.619

Cerón, M.C. (2006). Metodologías de la investigación social. Santiago de Chile: LOM. Ediciones.

Fernández, C. E., García, O. Y. \& López, R. T. (2015). Análisis factorial y confiabilidad del Cuestionario de Enfoques de Enseñanza. Revista Educación Médica del Centro, 7(4), 146-161.

Gallego, L. \& Araque, O. (2019). Variables de Influencia en la Capacidad de Aprendizaje. Un Análisis por Conglomerados y Componentes Principales. Información tecnológica, 30(2), 257-264.

García, G.M., Ayestarán, C. R., López, G. J. \& Tovar, V. M. (2019). Educar y formar al alumno talentoso: El afán de logro como competencia curricular. Comunicar, XXVII(60), 19-28. doi:https://doi. org/10.3916/C60-2019-02

García, M. C., López, V. O. \& Cabero, A. J. (2020). Autorregulación del aprendizaje en la Formación Profesional a Distancia: efectos de la gestión del tiempo. Revista de Educación a Distancia, 20(62).

Goodyear, P.\& Dimitriadis, Y.(2013). In media res: reframing design for learning. Research in Learning Technology, 21, 1-13.doi: http:// dx.doi.org/10.3402/rlt.v21i0.19909
Han, F. \& Ellis, R. (2020). Redes de aprendizaje personalizadas en contextos universitarios de aprendizaje semipresencial. Comunicar, (62), 19-30.

Hernández, G.A. (Coord.). (2017). Programa Indicativo para el Desarrollo de la Educación Superior en México: PIDESAD 2024 (ANUIES). Ciudad de México, México: SINED.

Hernández, R., Fernández, C. \& Baptista, P.(2014). Metodología de la investigación ( $6^{\mathrm{a}}$ ed.). Ciudad de México, México: McGraw-Hill.

Juca, M. F. J. (2016). La educación a distancia, una necesidad para la formación de los profesionales. Revista Universidad $y$ Sociedad, 8(1), 106-111.

Luna, E., Ponce, S., Cordero, G. \& CisnerosCohernour, E. (2018). Marco para evaluar las condiciones institucionales de la enseñanza en línea. Revista Electrónica de Investigación Educativa, 20(2), 114.doi: https://doi.org/10.24320/ redie.2018.20.2.2072

Maldonado, K. A. M. \& López, M. S. (2019). Satisfacción estudiantil universitaria: un referente para elevar los indicadores de los cursos en línea impulsados por la Coordinación General de Educación Virtual de la UAGro. Cuaderno de Pedagogía Universitaria, 16(31), 17-30.

Mestre, G. \& Ruíz, A., (2013). Modelos de educación a distancia. Bolivia: Bolivar.

Molina, G. A., Roque, L., Garcés B., Rojas, M. Y., Dulzaides, I. M. \& Selín, G. M. (2015). El proceso de comunicación mediado por las tecnologías de la información. Ventajas y desventajas en diferentes esferas de la vida social. MediSur, 13(4), 481-493.

Parra, M. (2015). Comparación de dos métodos de Análisis Factorial, de Componentes Principales, para determinar la Validez y Confiabilidad del Test de Estilos de aprendizaje de Kolb, en los estudiantes de Medicina, ULA, Mérida, 
Venezuela. Revista de Educación en Ciencias de la Salud, 12(1), 3.

Perch, J. S., Prieto, E. M., García, J. \& Orozco, E. (2019). Innovation and Practice in Education. Recuperado de https://www. researchgate.net/profile/Manuel_Prieto3/ publication/334098095_Innovation_ and_Practice_in_Education_2019/ links/5d164c12458515c11c00801c/ In novation-and-Practice-in Education-2019.pdf

Pulido, J. R., Rodríguez, J. A., Santana, M. G. \& Lorenzo, J. L. (2020). Aprendizaje del estudiante universitario: un estudio de caso. Educar, 56(1), 201-217.

Ramos, E. D., García, C. I., Sotelo, C. M., López, V. M. \& Murillo, P. L. (2020). Validación de un instrumento de estrategias para fortalecer el aprendizaje. Revista Electrónica Educare, 24(1), 1-15.

Rodríguez, R. Y. (2016). Elaboración de curvas de aprendizaje, para el ajuste del ciclo logístico, en la Planta de productos químicos de la Empresa LABIOFAM Villa Clara (Doctoral dissertation). Universidad Central "Marta Abreu" de Las Villas. Facultad de Ingeniería Mecánica e Industrial, Departamento de Ingeniería Industrial, Cuba.

Saza-Garzón, I. D. (2016). Estrategias didácticas en tecnologías web para ambientes virtuales de aprendizaje. Praxis, 12(1), 103-110.

Universidad Autónoma del Estado de México. (2010). Proyecto Curricular: Licenciatura en Negocios Internacionales. Estudio de factibilidad. Ciudad de México, México: UAEM.

Vargas, G. (2015). La formación virtual como herramienta de apoyo a los procesos formativos y no como reemplazo de la función docente. (Tesis doctoral). Universidad de Cartago, Panamá.
Zubieta, J. \& Rama, C. (2015). La educación a distancia en México: Una nueva realidad universitaria. Ciudad de México, México: Virtual Educa. 